\title{
Resource Scheduling using Cloud in Chemical \& Electro Chemical Coating Application
}

\author{
N.C. Brintha, J.T. Winowlin Jappes
}

\begin{abstract}
Due to the advancements and use of computer based technologies in manufacturing sectors, there has been a drastic change in how manufacturing industries perform their business. Manufacturing tied up with cloud computing technologies can help the customers, suppliers and manufacturers in several ways and hence can maximize profit in production lines. However, even though there are several policies for scheduling workflows in production, effective mapping of tasks with resources is always a challenging issue. If scheduling policies are inappropriate, it will have a negative impact on cost, time and therefore may affect the overall performance of the workflow. This work proposes a Multi-Objective Genetic Algorithm (MOGA) based scheduling model to schedule resources related to chemical and electro chemical coating. The major objective of this work is to reduce makespan, improve resource utilization and also minimize the overall cost of the workflow. This information obtained from the workflow can be used for better decision making when several tasks has to be done in parallel by migrating the tasks to the site of resource availability. The computational results also shows that MOGA performs well in makespan minimization, resource utilization and cost minimization because of its convergence speed and robustness. The analysis results prove that, MOGA can be optimal for estimating the path of where the work can be done such that the makespan and cost is minimized.
\end{abstract}

Keywords: coating, chemical process, Cloud computing, Manufacturing, Multi-Objective Genetic Algorithm (MOGA).

\section{INTRODUCTION}

$\mathrm{N}_{\text {ow-a-days, manufacturing industries distributed }}$ across several diversified locations, share product based information across production lines to maximize profit of manufacturing enterprises. In-order to promote information sharing and better utilization of resources, cloud based technologies may be incorporated in manufacturing industries. Cloud computing is a large scale distributed model in which resources are provisioned to the customers in a 'pay-as-you-go' model. The resources in cloud are heterogeneous, highly scalable, virtualized, configurable and reconfigurable [1]. Thus a cloud based manufacturing model supports resource sharing, rapid product development

Revised Manuscript Received on December 16, 2019

* Correspondence Author

N.C.Brintha*, Department of Computer Science and Engineering, Kalasalingam Academy of Research and Education, Tamilnadu, India. Email: n.c.brintha@klu.ac.in

J.T. Winowlin Jappes, Department of Mechanical Engineering, Kalasalingam Academy of Research and Education, Tamilnadu, India. Email: winowlin@klu.ac.in and delivery, cost minimization and better profit. Cloud Manufacturing incorporates resources that are geographically distributed as a centralized resource pool [2].

In manufacturing sectors, the resource demands of various jobs are not stable and they may fluctuate over time. A flexible scheduling system, dynamically allocates resources to tasks based on job's resource requirement. Scheduling manufacturing tasks and resources is considered as an NP hard problem [3]. Even though there are several intelligent optimization algorithms, Genetic Algorithm (GA) is found to be more effective in terms of resource provisioning and scheduling. In this work, optimal resource scheduling among jobs is considered as a main issue because this must be taken in to consideration to maximize profit. A multi objective resource scheduling mechanism based on genetic algorithm is proposed to improve cost and time. The usage of software decision making support will improve the productivity. MNC's are already supported with better support, but when considering Small and Medium Scale business, proper decision making is still at infancy. This is mainly due to the expense in implementing such systems, lack of trained personals and skilled labors.

In this investigation, plating methods based on chemical types is considered for coating on petrol engine cylinder block. In generally, chemical, electrochemical and mechanical methods are employed in plating of engine blocks. Even though corrosion resistant aluminium based alloys are used in casting of engine blocks, their tribological behaviour need to be enhanced. One of the very important aspects of chemical attack would be induced when, engine block under lubrication would be resisted by the protective coatings. Since many coating methods and many kinds of pre and post treatment are commercially available, the understanding of completion of work with minimum makespan, cost, etc. will be important for proper selection of coating methods. Moreover, thousands of Small and Medium Enterprises (SMEs) are available in these coating process hence it is necessary to maintain proper scheduling and information about the availability of resources that are identified with cloud concepts.

The rest of the paper is organized as follows: Section 2 focuses on the related works of scheduling manufacturing resources which is followed by the problem description depicted in Section 3. The resource scheduling by using Genetic Algorithm is described in Section 4 which is then followed by an example application study on manufacturing of four stroke petrol engine block focused 
towards coating and post operations in Section 5. Section 6, finally concludes the work.

\section{RELATED WORKS}

Cloud Manufacturing is a service oriented concept rather than the traditional production oriented method which incorporates several manufacturing resources through distributed networks [4]. Manufacturing industries mainly focus on time constrained product delivery with high quality and reduced cost. Normally, Small and Medium scale Enterprises (SMEs) can promote their product development by leasing resources from network rather than managing and maintaining all resources at the production site

In India, more than ten thousand SMEs are available for engine block manufacturing. One of the important processes in engine block manufacturing is coating which is used for enhancement of performances. In coating process, many methods like Chemical Coating [5], Electro-Chemical Deposition [6], Chemical Vapour Deposition [7], Diamond like Carbon Coating [8], etc., are available. Each methods have its own advantages. But, the cost of coating and time taken to complete the coatings are different. Based on the customer demands, like enhanced wear resistant, corrosion resistant, hardness, hot hardness, etc., different methods need to be selected. Mohammed et al. [17] proved that, modified genetic algorithm improves search accuracy and has better global convergence than other related algorithms.

In addition, pre and post treatment plays vital role in the performance of the produced components. Especially, case hardening treatments of engine block were selected by understanding the enhanced performance at the surface [9]. Many diffusion methods like carburizing [10] where carbon atoms are incorporated, diffusion of nitrogen in the case of nitriding [11] and combined inclusion of carbon and nitrogen in carbo-nitriding [12] are extensively used by the manufacturers. The hybridization of genetic algorithm with other algorithms are well suited for obtaining optimal solution [18].

With the combined tasks and multiple resources, it is necessary for a common platform to gather the pros and cons of resources and capability levels of each resources. By considering these concepts in a cloud system, the challenge in assigning and selecting optimum resources to complete the process with better output need to be analyzed. This investigation gives solution to manufacturers and customers for optimum scheduling of tasks and allocating the needed resources to tasks based on demand.

\section{PROBLEM FORMULATION}

The workflow application on chemical and electro chemical coatings on petrol engine block was considered and the various tasks and resources on the workflow was identified.

\section{A. Mapping of manufacturing tasks to Resources}

Different types of VM's are offered by IaaS cloud providers. An assumption is made that, the virtual machines have sufficient amount of storage to execute the tasks and the processing capacity of VM is measured in terms of Floating Point Operations Per Second (FLOPS). These information is used to calculate the execution time. The set of manufacturing tasks must be mapped to the available resources to carry out the necessary computation. The mapping of tasks with the centralized manufacturing resources in the resource pool is depicted in Fig.1.

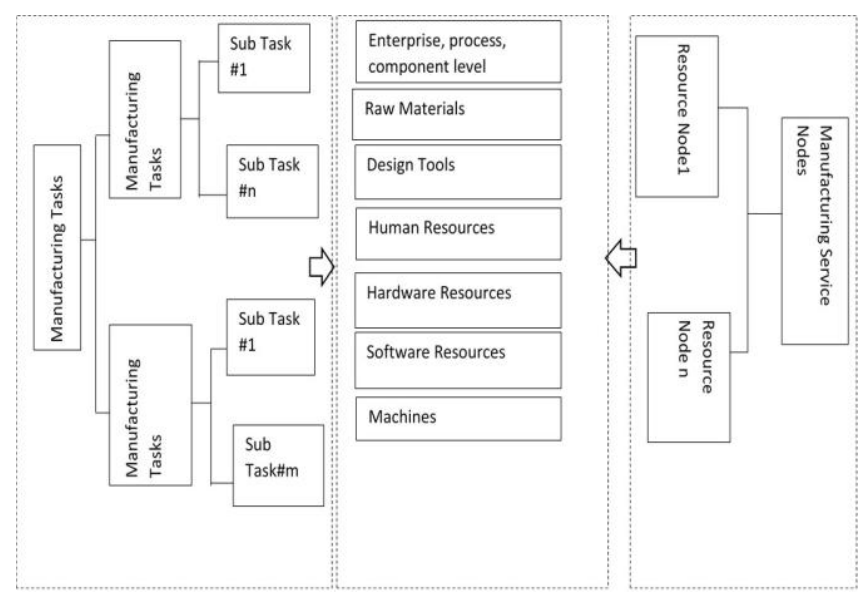

Fig. 1. Manufacturing Task - Resource Mapping

\section{B. Mathematical Modelling}

Consider a set of virtual machines $v m=v m_{1}, v m_{2}, \ldots, v m_{n}$ that are used to service the available tasks. Each virtual machine is considered to be parallel and independent and when a task starts its execution on a virtual machine it can continue without pre-emption. The major goal of this work is to reduce time and cost of execution.

The total completion time of each manufacturing task can be computed by summating the individual time of all subtasks MST. Total completion time of an individual task,

$$
\text { Time }_{T}=\sum_{i=1}^{j} M S T_{i j} \mid 1 \leq i \leq n ; 1 \leq j \leq m
$$

The overall job completion time or finish time is the total computational time of all tasks in the workflow.

$$
\text { Time }_{C T}=\sum_{\max } \text { Time }_{T_{i j}}
$$

where $i=1,2,3, \ldots, n$ represent the number of tasks in the workload and $j=1,2,3 \ldots, m$ represent the subtask within each task.

To promote execution of tasks, resources needed for task execution must be selected from virtualized servers. When a resource needed by a task is immediately available, it can start execution using the available resource otherwise the task must be moved to another site for execution. This adds 
transfer cost to the total computation cost during calculation. Thus the overall processing cost, 'Cost total' can be expressed using the total cost of all tasks and its transfer time if applicable.

$$
C_{T}=\sum_{n}\left(C_{\text {exe }}(i, j)+C_{\text {trans }}(j, K)\right) \text { for all } \mathrm{i}, \mathrm{j}, \mathrm{k} \in \mathrm{N}
$$

The objective function ${ }^{\prime} F^{\prime}$ is to minimize the cost and time of workload, so that the overall profit of production is maximized.

$$
\begin{aligned}
& F=\min \left(\begin{array}{c}
\text { Time }_{C T} \\
C_{W L}
\end{array}\right) \text { and Profit } \\
& \text { max } \\
& \text { Maximize } \operatorname{Res}_{U t i l}(t)=\frac{\sum_{i=1}^{m} R_{\text {actual }}(t)}{n \times C_{\text {Ser }}}
\end{aligned}
$$

Where ' $n$ ' denote the maximum number of servers, the number of VM placement is denoted by ' $m$ ' $\mathrm{m}, R_{\text {actual }}(t)$ is the capacity of resource allocated to a request at a particular time ' $r e q_{j}$ ' and the total capacity of each of the physical server ' $C_{\text {Ser }}$ '.

\section{MULTI-OBJECTIVE TASK SCHEDULING BASED ON GENETIC ALGORITHM (MOGA)}

This section describes a scheduling algorithm used for resource allocation in chemical and electro chemical deposition on engine block. The service model encompasses 4 tasks and 16 resources where the tasks and resources are dispersed across several sites. Genetic Algorithm (GA) is used as a search heuristic for identifying the optimal resource for a task that needs to be executed. This algorithm applies crossover, mutation and selection operators on the individual population to create effective schedules.

\section{A. Search Procedure using Genetic Algorithm}

Genetic algorithm is a heuristic search based procedure based on random probability. These are probabilistic optimization procedures which emulates the natural selection and evolution process. GA is a very commonly used optimization algorithm for finding the best job sequence for scheduling problems [13-15]. This starts with the generation algorithm which is based on the property of the survival of the fittest among the entire population. Once after the initial population is populated, an individual is chosen from every generation based on its fitness. A new set of population called offspring is generated using crossover and then perform mutation to attain better solution than the previous solutions which is then included in the new population.

Consequently, execution of Genetic Algorithm starts with a population of (typically random) chromosomes. Among the several individuals, one of the chromosome evaluates these structures. Then it allocates reproductive chances to represent an effective solution to the target problem. The best individuals among the population are provided with more of a set of individuals called population. It is an evolutionary

opportunities to reproduce than those which are poorer. The 'integrity' of the solution is typically specified by analyzing the present population.

The following things should be taken into consideration when assigning values to parameters for scheduling using the genetic algorithm.

1. Decision making on the size of population is an important issue. When the population size is too large, time consumption for finding the optimal solution among individuals will be more.

2. The recommended mutation rate of GA is about 0.5 to 1 percentage to obtain optimal results because the artificial process of mutation changes the numerical chromosome value. When mutation rates are fixed to smaller values which prevents the algorithm from local maxima and minima.

3. Genetic algorithms are usually subjected to premature convergence as a result of inappropriate crossover rate selection. Crossover rate may be selected with higher values i.e., 85 to 95 percentage for getting over this problem.

4. During the selection process, it is very important to choose the proper selection method for performing chromosome selection based on the application. Rank based and Roulette wheel method are found to be better, in comparison with several other selection methods.

\section{B. Allocation Strategy Based on GA}

\section{1) Encoding the population}

The encoding concept in genetic algorithm is used for transformation of problem space in to chromosomes. There are several forms of encoding such as tree encoding, binary encoding, and permutation encoding. In this work, binary encoding is used to generate candidates for the population and each candidates are represented as either 0 or 1 .In cloud manufacturing concept, a value 0 or 1 in the population corresponds to the manufacturing tasks. If the value is 0 , then the manufacturing task is not allocated to service node otherwise when the value is 1 , the manufacturing task is allocated to service node.

\section{2) Fitness Function Calculation}

The performance of the algorithm depends on the fitness value which represent the survival of the fittest in the population. Fitness function must be evaluated prior to applying crossover operation. In a multi-objective optimization problem it is very difficult to generate an optimal fitness value directly. So this model considers the most expected value and the least expected value to construct the fitness function. The difference between objective function and expected value is used to evaluate the effectiveness of this scheme. The overall flowchart for proposed GA based heuristic is as shown in Fig. 2. 


\section{Resource Scheduling using Cloud in Chemical \& Electro Chemical Coating Application}

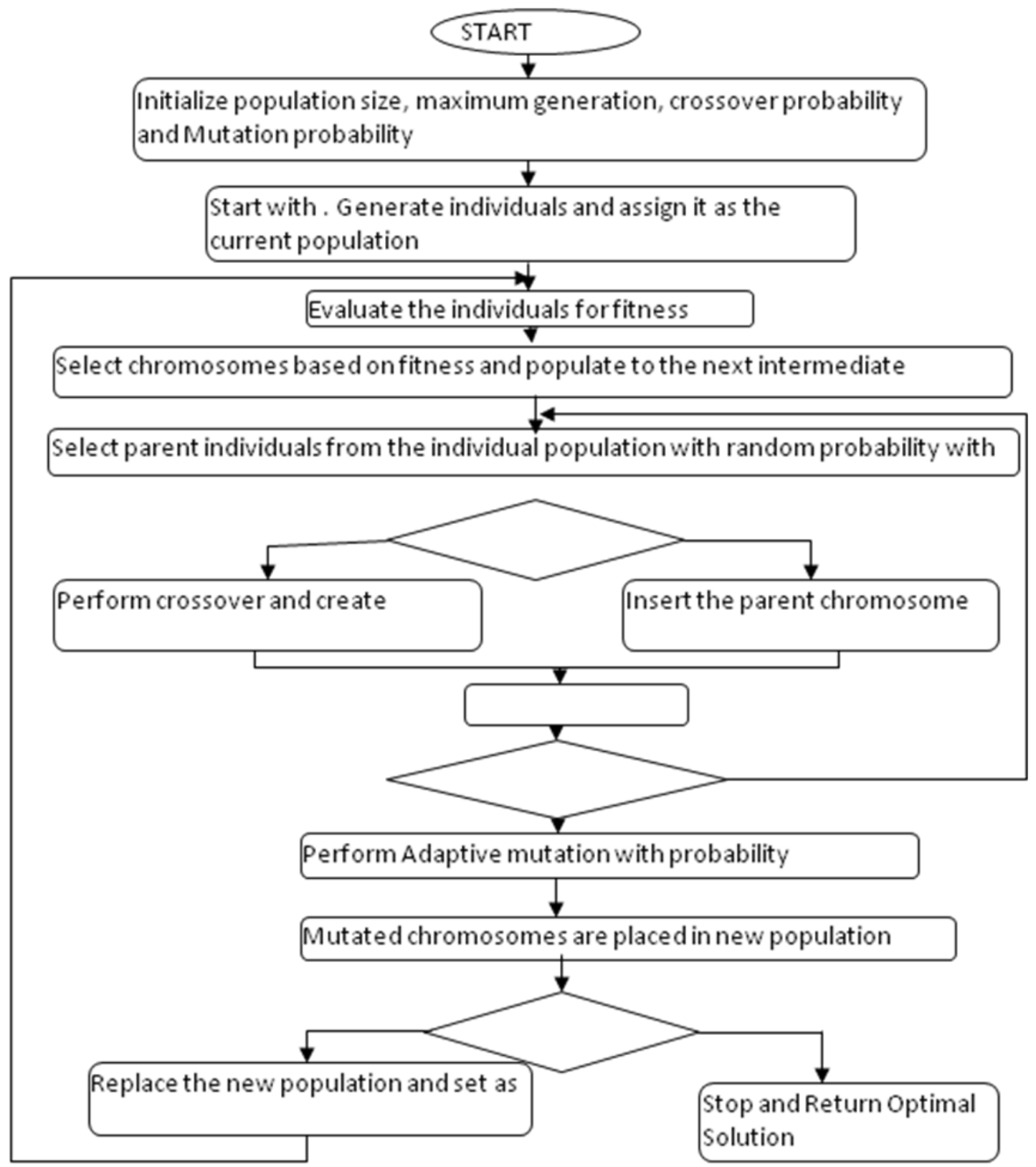

Fig.2. Scheduling Heuristic based on Genetic Algorithm

When the difference is smaller, the scheme becomes more effective. Based on the above analysis, the fitness evaluation function can be computed as in equation [5].The equation takes in to account the optimal value of two objective functions.

$$
\text { Fitness, } f=E_{\text {min }}=\sqrt{\left(E_{1}-E_{1}^{\prime}\right)^{2}+\left(E_{2}-E_{2}^{\prime}\right)^{2}}
$$

\section{3) Selection Operation}

A tournament based selection method is adopted to select the chromosomes from the population based on the fitness value. This selected individuals are then copied and made available for the next generation. Consider the population size as $P_{\text {Size }}$ and the fitness function as $f$, the probability of candidate selection $P_{\text {sel }}$ is represented as follows.

$$
P_{\text {sel }}=\frac{f}{\sum_{\text {Popsize }}}
$$

\section{4) Crossover Operation}

In this operation two strings are selected randomly with a crossover probability $P_{c}$ to produce a new offspring. The major objective of using crossover, is exchanging information between two strings to get a new offspring which is better than its 
parents. This work uses double point crossover strategy to generate offspring. This method randomly selects two gene segments, and exchanges gene among the two parent individual.

\section{5) Mutation Operation}

Mutation is a process which adds new information to the search and avoid the individuals from getting trapped on local optimal solutions. From the individual population calculate the mutation probability $P_{m}$. Set up a threshold value and when the probability is greater than the threshold, a gene segment is selected randomly from the population. In the selected gene segment one of the gene is changed to 1 and all the other genes in the same segment are initialized to 0.

\section{Schedule Generation by Mapping Resources and Tasks}

The particles in the population are analyzed and the position of each individual in the population has to be converted to appropriate schedule. First, the resources has to be identified for task migration. The execution time and cost of the workload is set as zero. Then, MOGA algorithm used to estimate the execution time of the workflow on each resources

Initially, the task-resource mapping must be made and the value of start time is determined on the basis of some scenarios. When a task has no preliminary stages, it can start its execution immediately when the resource is available.
But, when the requested resource is busy or unavailable, the task is allowed to wait for its execution until the VM is available.

\section{RESULTS AND DISCUSSIONS}

In this investigation, experimentation was carried out on a case study of four stroke petrol engine block focused towards coating and post operations. Chemical and electro chemical coatings play a major role in performance enhancements of engine blocks. From the SMEs point of view, their limited resources need to be explored in a perfect domain to attract many customers. From the customer point of view, availability of resources with enhanced performances at limited cost with timely completion of work is expected.

In the present work, four tasks namely Pre-Treatment, Coating Process, Case hardening and Non Destructive Inspection are selected which are essential part of operations in the manufacturing of engine blocks. Each tasks could be competed with the help of four resources which are listed in Table I. Simulations were carried out on cloudsim software and the algorithm was integrated with the cloudsim layer. The tasks and resources were identified as in Table I and for processing each tasks it should be mapped to the corresponding VMs.

Table- I: Identified Task-Resource Set

The virtual machines are distributed across several locations across the country and these locations are the SMEs which hosts the resources for servicing the tasks.

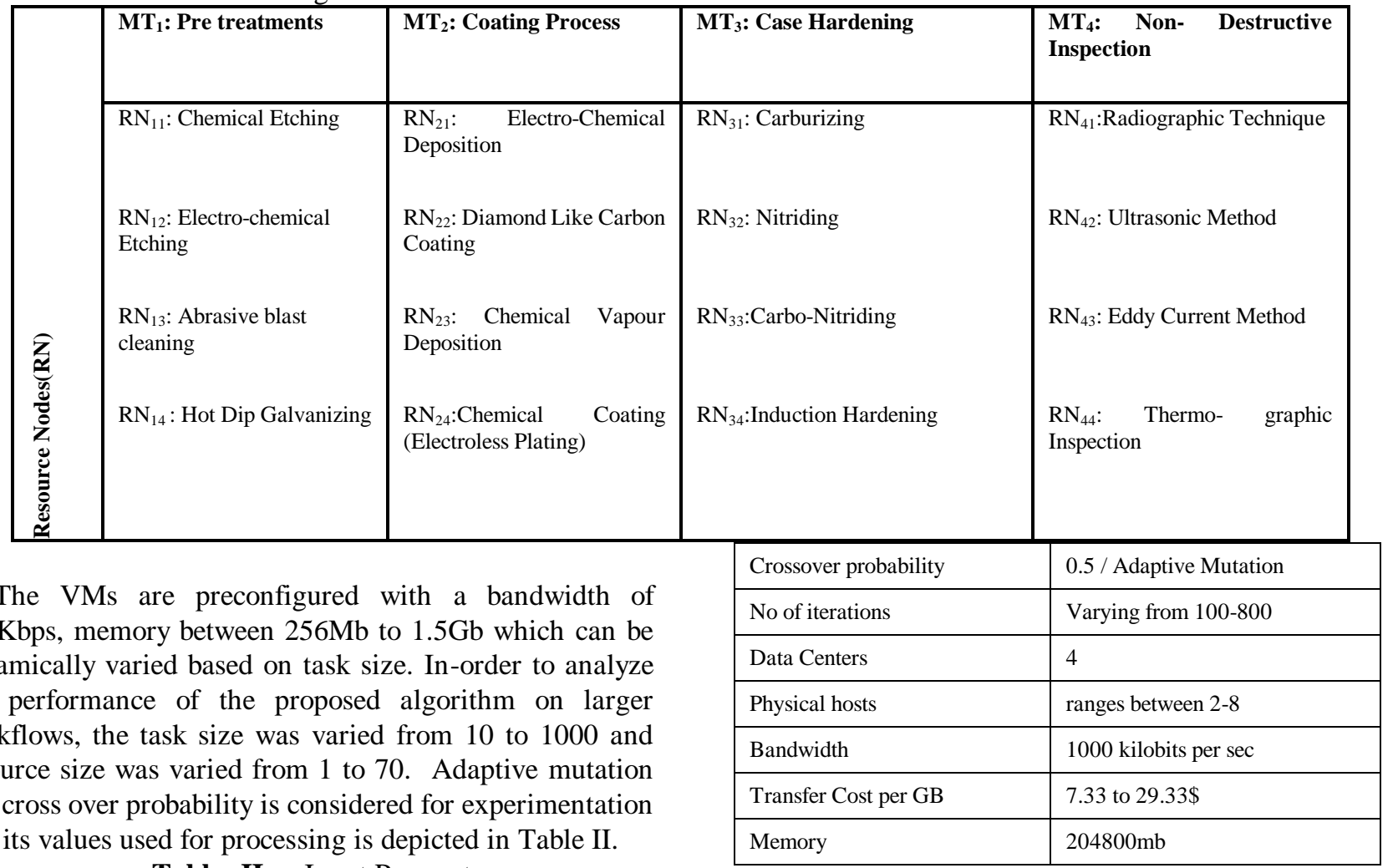
and its values used for processing is depicted in Table II.

Table- II: Input Parameters

\begin{tabular}{|l|l|}
\hline Parameters & Values \\
\hline Mutation probability & $0.6 /$ Adaptive Mutation \\
\hline
\end{tabular}




\section{Resource Scheduling using Cloud in Chemical \& Electro Chemical Coating Application}

This work explores the possibilities of utilizing the chemical and electro chemical methods of pre-treatment, coating and post treatment. The chemistry and electro chemistry behind this deposition methods modifies the bonding phenomena, and there-by improves the workability. By considering chemical way of deposition which is normally done through electroless process, gives semi crystalline nature of deposits. These deposits have remarkable benefit when surface treated through carbo-nitriding. In electro-chemical deposition methods, electro plating produces ions which gets coated. In a coated condition, the hardness value could be improved.

Unlike the mentioned deposition methods, an advanced Chemical Vapour Deposition (CVD) process produces thin (may in nano-scale also) coating with high hot hardness. But the drawback in all the strategies taken in to consideration will be cost. In addition, prep and post treatment which is to be employed will have considerable influence on processing time, cost, etc.

Further all the four suggested non-destructive inspection methods are considered to perform well for all flaws. By considering all complexities in the process and availability of resources, identification of resources to complete the task is taken as case study in this work .So this approach using genetic algorithm takes in to account the objective of optimal scheduling to minimize cost and time.

\section{A. Effect of Cost Minimization}

The optimal solution using genetic algorithm for task-resource mapping is 010100010100100010 in Table III. In this representation among the three available resources in each resource types, the manufacturing task $M T_{1}$ was assigned to the second instance of resource $R_{1}$; the second casting task $M T_{2}$ was assigned to the first instance of resource $R_{2}$; the third casting task $M T_{3}$ was assigned to the second instance of resource $R_{3}$; the fourth casting task $M T_{4}$ was assigned to the first resource instance of resource $R_{4}$; the next casting task $M T_{5}$ was assigned to the first resource instance of resource $R_{5}$ and the last casting task $M T_{6}$ was assigned to the second resource instance of resource $R_{6}$.

Table- III: GA Based Task-Resource Mapping for the Casting Workflow

\begin{tabular}{|c|c|c|c|c|c|c|c|c|c|c|c|c|c|c|c|c|}
\hline \multicolumn{3}{|c|}{$\mathbf{R}_{1}$} & \multicolumn{3}{|c|}{$\mathbf{R}_{2}$} & \multicolumn{3}{|c|}{$\mathbf{R}_{3}$} & \multicolumn{3}{|c|}{$\mathbf{R}_{4}$} & \multicolumn{2}{|c|}{$\mathbf{R}_{\mathbf{5}}$} & \multicolumn{3}{|c|}{$\mathbf{R}_{6}$} \\
\hline$R_{11}$ & $\mathbf{R}_{12}$ & $\mathbf{R}_{13}$ & $\mathbf{R}_{21}$ & \begin{tabular}{|l|l}
$\mathbf{R}_{22}$ & $\mathbf{R}$ \\
\end{tabular} & $\mathbf{R}_{23}$ & $R_{31}$ & \begin{tabular}{|l|l}
$\mathbf{R}_{32}$ & $\mathbf{R}$ \\
\end{tabular} & $\mathbf{R}_{33}$ & $R_{41}$ & \begin{tabular}{l|l}
$\mathbf{R}_{42}$ & $\mathbf{R}$ \\
\end{tabular} & $\begin{array}{ll}\mathbf{R}_{43} & \mathbf{R} \\
\end{array}$ & \begin{tabular}{l|l}
$\mathbf{R}_{51}$ & $\mathbf{R}$ \\
\end{tabular} & \begin{tabular}{l|l}
$R_{52}$ & $R$ \\
\end{tabular} & $\mathbf{R}_{6}$ & $\mathbf{R}_{62}$ & $\mathbf{R}_{63}$ \\
\hline & 1 & 0 & 1 & \begin{tabular}{l|l}
0 & 0
\end{tabular} & 0 & 0 & \begin{tabular}{l|l}
1 & 0
\end{tabular} & 0 & \begin{tabular}{l|l}
1 & 0
\end{tabular} & \begin{tabular}{l|l}
0 & 0
\end{tabular} & \begin{tabular}{l|l}
0 & 1
\end{tabular} & \begin{tabular}{l|l}
1 & 0
\end{tabular} & 0 & 0 & 1 & 0 \\
\hline
\end{tabular}

Processing Cost of task on resource ${ }^{\prime} C_{r}{ }^{\prime}: \$ 341.22$

Transfer Cost for the current workflow ${ }^{\prime} C_{\text {Trans }}{ }^{\prime}: \$ 7.42$

Total Cost of the casting workflow ${ }^{\prime} C_{T}{ }^{\prime}: \$ 348.64$

\section{B. Effect of Makespan Minimization}

During the process, larger mutation and crossover probabilities are considered at the early stages which is then reduced during the later stages for achieving greater convergence. The convergence rate of this algorithm was found to be better when the generations are 115, 62 and 98. The population size $P_{\text {size }}$ was varied based on the workflow size and the number of generation $G_{\max }$ was taken as 150 . The error rate for the workflow is $18 \%$ and standard deviation was 0.3875 .

\section{1) Impact of Makespan by varying Tasks}

The performance of the casting workflow was evaluated based on makespan by varying the number of virtual machines 10, 20 and 30. The increase in the number of manufacturing tasks affects the execution time of jobs i.e. increasing the number of tasks proportionally maximizes the makespan which is described in Fig. 3.

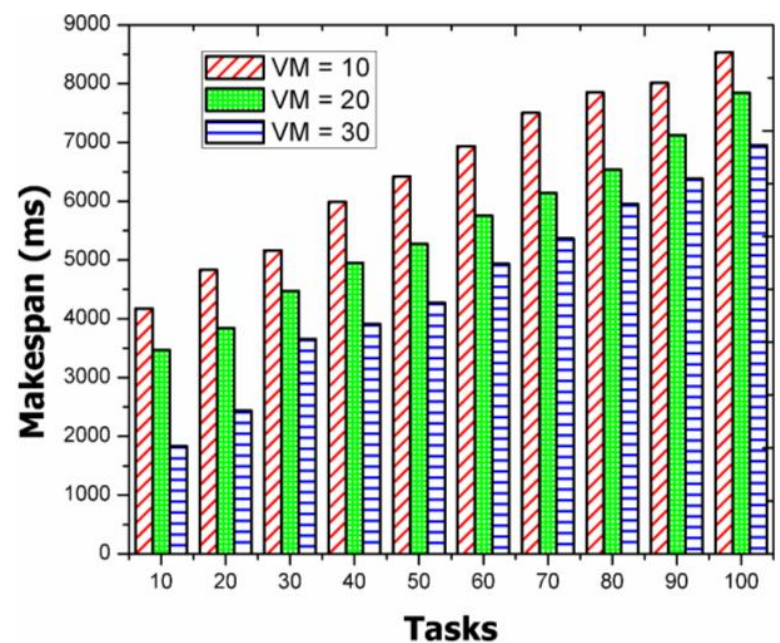

Fig. 3. Impact of GA on makespan computation by varying the number of tasks

When the number of virtual machine is less, unavailability of resources to service the tasks increases the makespan. Thus, when the number of VM was found to be 10 , the makespan after execution of 100 manufacturing tasks was $8537 \mathrm{~ms}$. But when the number of VM was increased by 20 , there was a decrease in makespan of $7845 \mathrm{~ms}$ and further increase in makespan results in about $6952 \mathrm{~ms}$. Thus, provisioning more VMs to manufacturing tasks enables completion of the task within the minimum deadline.

2) Impact of Makespan by varying Resources

Simulation was carried out by considering constant task size of 50,100 and 150. By considering the above workload, the resources were distributed across SMEs in several regions. When the number of resources in the workflow was 5, the makespan was found to be $8014 \mathrm{~ms}$ for a workload of 50 tasks. But when the number of resources was increased to 70 VMs, the reduction in makespan was drastic and found to be $2843 \mathrm{~ms}$. When considering the task size as 100 and the number of VMs as 5 the makespan was found to be $8952 \mathrm{~ms}$ but increase in VM to 70 resulted in the reduction in makespan 3383ms. Fig. 4 depicts that, increasing the task size increases makespan but it can be compromised by providing more resources thereby increasing resource availability.

\section{Published By:}




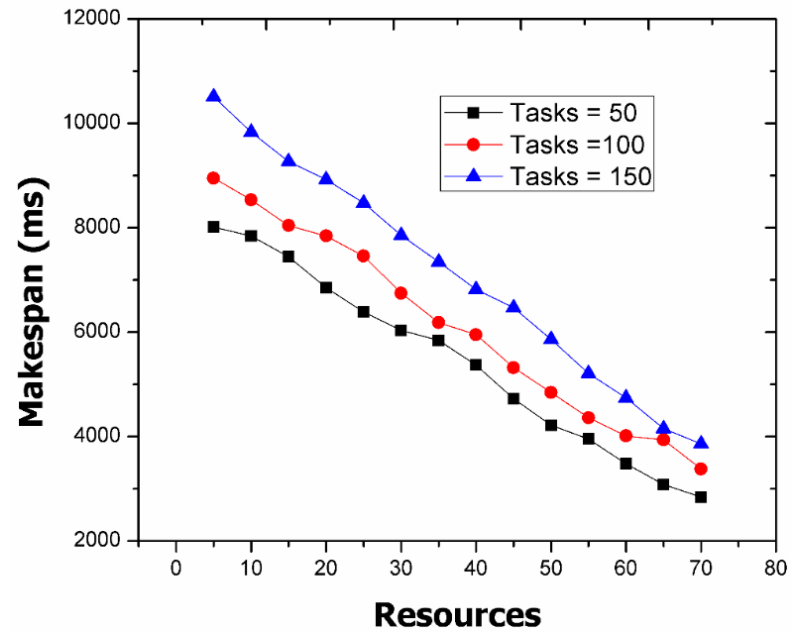
Count

\section{3) Impact of Service Node Replication on Makespan Computation}

When the number of service nodes or data centers is increased, makespan is reduced because of the availability of more resource providers to service the tasks. The resource identified on casting workflow is considered for the simulation process and these resources are replicated across multiple sites. The performance of workflow is evaluated based on this replicated and non-replicated resource sites.

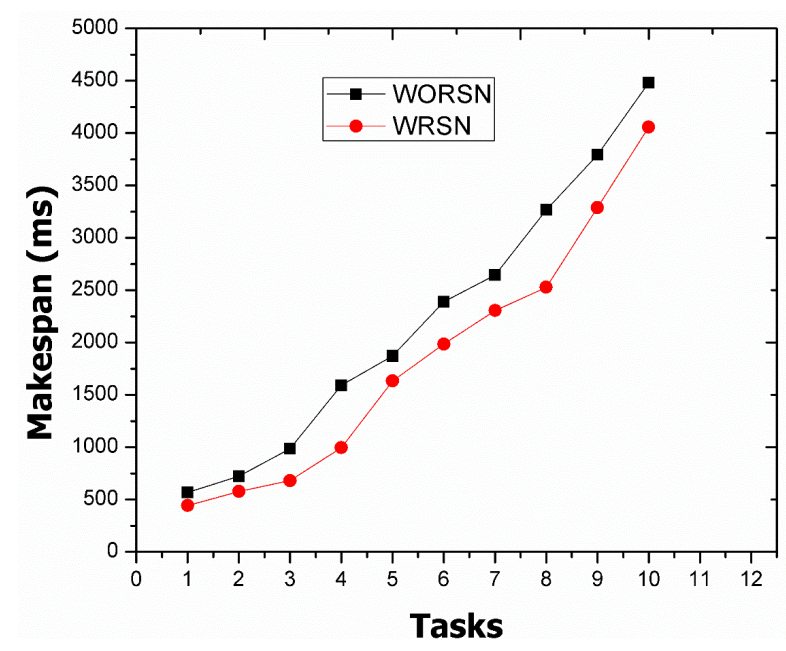

Fig.5. Impact of GA on Makespan with respect to replicated and non-replicated Service Nodes

After evaluation through 600 iterations, the resultant performance of the workflow is depicted in Fig. 5. The simulation results shows that, there is an improvement in makespan when the resource is replicated across multiple sites rather than considering the workflow without replication. When considering the first task on the work load, makespan is found to be $567 \mathrm{~ms}$ and for last task it is $4483 \mathrm{~ms}$ by considering the distribution without replicated service nodes. But when the resources are replicated across multiple sites, there is further improvement in the performance of the workflow. The first task is found to complete within $443 \mathrm{~ms}$ and the last task completes by about
Fig.4. Impact of GA on makespan by increasing Resource

$4058 \mathrm{~ms}$. From these performance results it is found that, when the resource nodes are replicated across several sites there is an improvement in makespan.

\section{CONCLUSION}

This work presents a resource management and decision making strategy based on genetic algorithm for addressing the problem of cost and time of mapping tasks to resources in Small and Medium Scale Business (SMEs). The principles of IaaS cloud is used in this work, to support elasticity, heterogeneity and dynamicity. A multi-objective job scheduling framework based on cloud is considered for coating and post operations on four stroke petrol engine block. The computations prove the effectiveness of the algorithm using the proposed approach also the obtained results shows that genetic algorithm is more robust and also provides rapid convergence to achieve optimal results. So this approach can support in better decision making for SME's to select the better path for migrating tasks, such that, the overall cost and time of production is minimized. As a future work, comparison on the performance of workload will be evaluated. This approach provides a decision making support system, which can be further extended for supporting large scale firms by taking private and public cloud in to consideration.

\section{REFERENCES}

1. Dazhong, W, Matthew, J.G., David, W, Rosen and Dirk Schaefer., "Cloud manufacturing: Strategic vision and state-of-the-art", Journal of Manufacturing Systems, Vol.32, No.4, 2013, pp.564-579.

2. B.H. Li, L. Zhang, S.L.Wang, F. Tao, J.W. Cao, X.D. Jiang, X. Songand, X.D Shai., "Cloud manufacturing: a new service-oriented networked manufacturing model", Computer Integrated Manufacturing Systems, vol. 16, no. 1, 2010 pp.1-7

3. M. R. Garey and D. S. Johnson, Computer and Intractability: A Guide to the NP-Completeness, vol. 238, New York, NY, USA:Freeman, 1979.

4. C. Yin, B.Q. Huang, F.Liu, L. J.Wen, Z.K. Wang, X.D. Li, S.P. Yang, D. Yeand X.H. Liu, "Common key technology system of cloud manufacturing service platform for small and medium enterprises", Computer Integrated Manufacturing Systems, vol. 17,no. 3, (2011), pp.77-84.

5. Dexin Chen, Yang Zhang, Takeshi Bessho, Jing Sang, Hidetoshi Hirahara, Kunio Mori, Zhixin Kang, "Layer by layer electroless deposition: An efficient route for preparing adhesion-enhanced metallic coatings on plastic surfaces", Chemical Engineering Journal, vol. 303, 1 November 2016, pp. 100-108.

6. M. Manna, M. Dutta, "Effect of prior electro or electroless Ni plating layer in galvanizing and galvannealing behavior of high strength steel sheet", Surface and Coatings Technology, In Press, Accepted Manuscript, Available online 22 December 2016.

7. T. Schmauder, K.-D. Nauenburg, K. Kruse, G. Ickes, "Hard coatings by plasma CVD on polycarbonate for automotive and optical applications", Thin Solid Films, vol. 502, no.1-2, 28 April 2006, pp. 270-274.

8. Dobrenizki, L., Tremmel, S., Wartzack, S., Hoffmann, D.C., Brögelmann, T., Bobzin, K., Bagcivan, N., Musayev, Y., Hosenfeldt, T., "Efficiency improvement in automobile bucket tappet/camshaft contacts by DLC coatings - Influence of engine oil, temperature and camshaft speed", Surface and Coatings Technology, vol. 308, 25 December 2016, pp.360-373.

9. Cuesta, I.I., Alegre, J.M., "Hardening evaluation of stamped aluminium alloy components using the Small Punch Test, Engineering Failure Analysis", vol. 26, December 2012, pp.240-246.

10. Zhi-kai Chen, Ti Zhou, Ruo-yu Zhao, Hai-feng Zhang, Shu-chao Lu, Wan-shi Yang, Hong Zhou, "Improved fatigue wear resistance of grey cast iron by localized laser carburizing", Materials Science and Engineering: A, vol. 644, 17 September 2015, pp. 1-9.

11. Naeem, M., Shafiq, M., Zaka-ul-Islam, M., Ashiq, A., 


\section{Resource Scheduling using Cloud in Chemical \& Electro Chemical Coating Application}

Díaz-Guillén, J.C., Shahzad, M., Zakaullah, M., "Enhanced surface properties of plain carbon steel using plasma nitriding with austenitic steel cathodic cage", Materials \& Design, vol. 108, 15 October 2016, pp. 745-753

12. Manoj Mathew, P.K. Rajendrakumar, Optimization of process parameters of boro-carburized low carbon steel for tensile strength by Taquchi method with grey relational analysis, Materials \& Design, vol. 32, no. 6, June 2011, pp. 3637-3644

13. Chen, Z. G., Du, K. J., Zhan, Z. H. and Zhang, J., "Deadline constrained cloud computing resources scheduling for cost optimization based on dynamic objective genetic algorithm". In Proceedings of the IEEE Congress on Evolutionary Computation, 2015.

14. Ge, J. W. and Yuan, Y. S., "Research of cloud computing task scheduling algorithm based on improved genetic algorithm," in Applied Mechanics and Materials, vol. 347-350, no.4, 2013, pp. 2426-2429.

15. Shi Chen, Jie Wu, Zhihui $\mathrm{Lu}$, "A Cloud Computing Resource Scheduling Policy Based on Genetic Algorithm with Multiple Fitness", 2012 IEEE 12th International Conference on Computer and Information Technology.

\section{AUTHORS PROFILE}

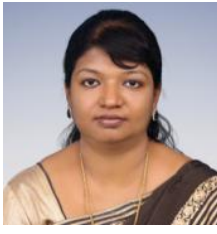

Brintha N C graduated in 2002 from Manonmaniam Sunderanar University, India. In 2006, she received her M.E Degree with distinction in Computer Science and Engineering from A.K.C.E, Anna University, Chennai. She completed her Ph.D degree in 2018 in the area of Cloud Manufacturing under Anna University, Chennai. Currently, she is working as Associate professor/ CSE in Kalasalingam Academy of Research and Education, India. Her research interests include Cloud computing, Optimization, Scheduling, Cloud Manufacturing and so forth.

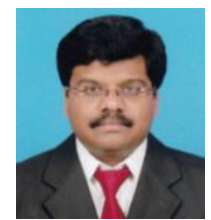

Winowlin Jappes $\mathbf{J} \quad \mathbf{T}$ graduated in 1997 from Manonmaniam Sunderanar University, India in Mechanical Engineering. In 1999, he has completed his masters in Production Engineering from Annamalai University. He completed his Ph.D degree in 2004 in the area of Composite Deposition at Indian Institute of Technology Madras, Chennai. He has completed three DST funded research projects and published more than 125 research articles which includes 65 International Journal papers. Currently, he is working as Senior Professor in Kalasalingam University, India. His research interests includes high performance composite materials, machining of hard materials, optimization techniques, etc. At present, he is working as Professor and Dean, School of Automotive and Mechanical Engineering, Kalasalingam Academy of Research and Education, India.
16. Mohammed Abdullahi, Md Asri Ngadi, Salihu IdiDishing, Shafi, "An efficient symbiotic organisms search algorithm with chaotic optimization strategy for multi-objective task scheduling problems in cloud computing environment", Journal of Network and Computer Applications, vol. 133, 1 May 2019, pp. 60-74.

17. DivyaChaudhary, BijendraKumar, Cost optimized Hybrid Genetic-Gravitational Search Algorithm for load scheduling in Cloud Computing, Applied Soft Computing, vol. 83, October 2019, https://doi.org/10.1016/j.asoc.2019.105627 